\title{
Cavity QED and quantum-information processing with "hot" trapped atoms
}

\author{
L.-M. Duan, ${ }^{1, *}$ A. Kuzmich, ${ }^{2}$ and H. J. Kimble ${ }^{2}$ \\ ${ }^{1}$ Institute for Quantum Information, MC 107-81, California Institute of Technology, Pasadena, California 91125-8100 \\ ${ }^{2}$ Norman Bridge Laboratory of Physics 12-33, California Institute of Technology, Pasadena, California 91125
}

(Received 12 September 2002; published 17 March 2003)

\begin{abstract}
We propose a method to implement cavity QED and quantum-information processing in high- $Q$ cavities with a single trapped but nonlocalized atom. The system is beyond the Lamb-Dicke limit due to the atomic thermal motion. Our method is based on adiabatic passages, which make the relevant dynamics insensitive to the randomness of the atom position with an appropriate interaction configuration. The validity of this method is demonstrated from both approximate analytical calculations and exact numerical simulations. We also discuss various applications of this method based on the current experimental technology.
\end{abstract}

DOI: 10.1103/PhysRevA.67.032305

PACS number(s): 03.67.-a, 42.50.Gy

\section{INTRODUCTION}

Trapping of single atoms in high- $Q$ cavities opens up exciting possibilities for the observation and manipulation of the dynamics of single particles and for control of their interactions with single-mode photons $[1,2,4,5]$. Such possibilities could have wide applications, such as for the generation of nonclassical or entangled optical pulses [6,7], for observing strong cavity-QED effects $[4,5,8]$ and, more remarkably, for implementation of quantum communication and computation [9-13]. The trapping potential for confining single atoms can be created by diverse avenues, including by the cavity-QED light itself $[4,5]$, by additional far-offresonant trapping (FORT) beams [2], and by combining single trapped ions with high-finesse optical cavities [14,15]. In this paper, we will direct our attention principally to trapping in cavity QED by way of an additional FORT beam, although our results are applicable to broader settings.

The first experiment to achieve strong coupling in cavity QED with trapped atoms was that of Ref. [2], which employed an intracavity FORT beam and reported trapping lifetimes of $28 \mathrm{~ms}$. By now, this experiment has attained much longer trapping times, with recent work demonstrating lifetimes in excess of $1 \mathrm{~s}[3,16]$. By contrast, atomic localization by way of the cavity-QED field itself has led to trapping within a single axial well with mean trapping time $\tau$ $\approx 340 \mu \mathrm{s}$ [4] and to localization across many axial wells with mean time $\tau \approx 280 \mu$ s [5].

The long trapping times achieved with an intracavity FORT beam set the stage for diverse applications in quantum-information science, which motivates the current analysis. However, one of the main obstacles to the experimental demonstration of these applications is that the position of the trapped atom is not well fixed within the cavity. The coupling rate $g$ between the atomic internal levels and the cavity mode depends on the atom's position $\mathbf{r}$ through the relation

$$
g(\mathbf{r})=g_{0} \chi(\mathbf{r})
$$

\footnotetext{
*Email address: 1mduan@caltech.edu
}

with the mode function

$$
\chi(\mathbf{r})=\sin \left(k_{0} z\right) \exp \left[-\left(x^{2}+y^{2}\right) / w_{0}^{2}\right],
$$

where $g_{0}$ is the peak coupling rate, $w_{0}$ and $k_{0}=2 \pi / \lambda_{0}$ are, respectively, the width and the wave vector of the Gaussian cavity mode, and $z$ is assumed to be along the axis of the cavity. Due to the randomness of the atom's position $\mathbf{r}$, we have an unknown randomly changing coupling rate $g(\mathbf{r})$. Most of the applications of this setup assumed a fixed known coupling rate $g$. Therefore, before the experimental demonstration of these schemes, first one needs to solve the problem associated with the random coupling.

Intense experimental efforts have been taken to localize the atom inside the cavity so as to fix the coupling rate $g(\mathbf{r})$, with notable recent success attained via ion traps $[14,15]$. In the cavity-QED experiments employing cold atoms and without FORT beams $[1,17,18]$, atoms were dropped through the cavity and followed random trajectories with large axial heating. As a result, the magnitude and the sign of $g(\mathbf{r})$ were not well controlled. With a FORT beam and with current experimental capabilities [2,3,16], an atom can be trapped inside one potential well along the cavity axis with a fixed sign of $g(\mathbf{r})$. But the atom still has appreciable kinetic energy and is not fully localized, leading to significant variations in the magnitude of the coupling rate $g(\mathbf{r})$.

The randomness of the coupling rate $g(\mathbf{r})$ comes from several contributions: first, the trapped atom is still quite hot in the current experimental setup. Its kinetic energy from the thermal motion is typically lower but not much lower than the depth of the trapping potential. The atom's oscillation amplitude $d$ in the trap is comparable to the optical wavelength $\lambda_{0}$, so it does not satisfy the usually assumed LambDicke condition $d \ll \lambda_{0}$. Due to the thermal motion of the atom, the coupling rate $g(\mathbf{r})$ typically has a variation within a factor of 2 with the current experimental technique. Certainly, the atom will become better localized as cooling techniques are adapted to cavity QED and its energy is reduced $[19,20]$. However, due to the presence of the cavity and the trapping potential, it is still experimentally hard to achieve efficient cooling inside the cavity [19-21]. Furthermore, even if we assume that the atom has been precooled and localized initially to the Lamb-Dicke limit, the implemented 
application protocols will still tend to heat the atom due to photon recoils from the spontaneous emissions [22,23]. As a result of the heating, the atom may go out of the Lamb-Dicke limit after a short time. Finally, even if we neglect all the motional and the heating effects of the trapped atom, there is still some uncertainty of the coupling rate. The intracavity field of the FORT beam forms many potential wells inside the cavity, and in current experiments, one cannot control and does not know precisely in which well the atom is trapped. The FORT beam has a wavelength $\lambda_{F}$ different from the cavity-QED wavelength $\lambda_{0}$, so, even if the atom is kept very cold and well localized at the bottom of the trapping potential well, we still might not know exactly the coupling rate, since the bottoms of different potential wells have different coupling rates [24].

Here, to overcome these difficulties, we propose a method to do cavity-QED and quantum-information processing directly with hot atoms with an inhomogeneous distribution in position and/or a time-varying location. The method is based on adiabatic passages with a new interaction configuration. Adiabatic passages have been studied in the context of cavity QED [6,13,26,27], and have been adopted in some recent experiments $[17,18]$. Normally, schemes based on adiabatic passages are more insensitive to certain parameter changes compared with the corresponding Raman schemes. Some initial indication of insensitivity of the adiabatic passage scheme to certain parameter changes was already illustrated in Ref. [27] for a certain cavity-QED scheme. However, to make the whole system dynamics insensitive to variations of the coupling rate $g(\mathbf{r})$, the direct use of the usual adiabatic passage schemes is not enough to achieve this goal, and we also need to design a different and appropriate interaction configuration. The relevant dynamics of adiabatic passages are determined by the relative ratio between different coupling rates, and are almost independent of their absolute values. Thanks to this property, with an appropriate design of the interaction configuration, we can make different coupling rates have the same dependence on the atom's position $\mathbf{r}$, and, therefore, the system dynamics, determined by their relative ratios, will become independent of $\mathbf{r}$. As a result, though the atom's position may be unknown and time dependent, the output signal from the cavity is still controllable and has definitely known properties. This is the difference between the scheme here and the usual adiabatic passage schemes $[13,17,18,27]$. Note that the method described here is also different from some previous quantum computation schemes with hot trapped ions $[28,29]$, where the LambDicke condition is still required.

The paper is arranged as follows: In Sec. II, we explain the basic idea of the method, and then describe and solve the model Hamiltonian analytically following some well-known approach based on the adiabatic approximation. This approximate analytical approach is still not enough to fully understand the experiments, so in Sec. III, we give an exact numerical simulation of the model, with the emphasis on checking the validity of the introduced approximations and calculating various kinds of noise magnitudes relevant for the on-going experimental efforts. The calculations show that we can get reasonably good signal-to-noise ratios with typi-
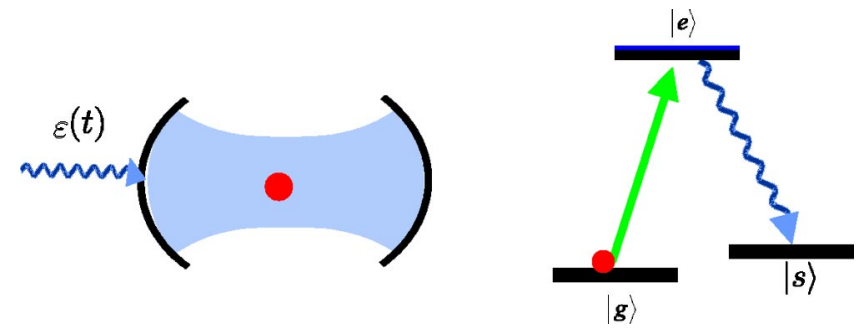

FIG. 1. Schematic setup. Left side: a single atom trapped in a high- $Q$ cavity, which is driven by a classical laser pulse $\varepsilon(t)$. Right side: the relevant atomic level structure.

cal experimental values for the parameters. In Sec. IV, we briefly review some known cavity-QED and quantuminformation processing schemes, and then discuss how to incorporate the present method into these schemes to improve their performance against the randomness in the atom's position. After this incorporation, with hot nonlocalized atoms, one can still realize many kinds of cavity-QED and quantum-information processing schemes, including, for instance, the controllable single-photon or entangled-photon source, quantum communication between cavities, atomic entanglement generation, teleportation, and Bell inequality detection. Section V gives a synopsis of parameters relevant to our current experiment for a single-atom trapping with a FORT beam at Caltech $[2,3,16]$. We summarize the results in the final section.

\section{CAVITY QED WITH A NONLOCALIZED TRAPPED ATOM: THE SCHEME}

\section{A. Basic idea}

First, we explain the basic idea of this method by considering a single trapped atom, which has three effective levels $|g\rangle,|e\rangle,|s\rangle$, as shown in Fig. 1. The two ground states $|g\rangle$ and $|s\rangle$ can correspond, for instance, to sub-Zeeman levels in the $F=3$ and $F=4$ manifolds, respectively, for the cesium atom. The transition $|e\rangle \rightarrow|s\rangle$ is coupled resonantly to the cavity-QED mode $a$ with a coupling rate $g(\mathbf{r})$ in the form of Eq. (1). A classical laser field $\varepsilon(t)$ incident from one mirror of the cavity (see Fig. 1) drives the transition $|g\rangle \rightarrow|e\rangle$ through another cavity mode $a^{\prime}$. We assume for simplicity that $a$ and $a^{\prime}$ have the same spatial mode structure with the same frequency (for example, they can be of different polarizations) [25]. The driving laser $\varepsilon(t)$ is resonant to the transition $|g\rangle \rightarrow|e\rangle$, so it is far-off-resonant to the cavity mode $a^{\prime}$ with a large detuning $\omega_{g s}$, where $\omega_{g s}$ denotes the splitting between the levels $|g\rangle$ and $|s\rangle$. Due to the off-resonant driving by $\varepsilon(t), a^{\prime}$ can be described classically by its mean value $\left\langle a^{\prime}\right\rangle=\alpha(t) e^{-i \omega_{g e} t}$ ( $\omega_{g e}$ is the frequency splitting between the levels $|g\rangle$ and $|e\rangle$ ), which couples resonantly to the transition $|g\rangle \rightarrow|e\rangle$ with a Rabi oscillation frequency $\Omega(\mathbf{r}, t)$. Since $a$ and $a^{\prime}$ have the same spatial mode structure, the Rabi frequency $\Omega(\mathbf{r}, t)$ will depend on the atom's position $\mathbf{r}$ by the same mode function $\chi(\mathbf{r})$, i.e., $\Omega(\mathbf{r}, t)$ can be factorized as

$$
\Omega(\mathbf{r}, t)=\Omega_{0}(t) \chi(\mathbf{r})=r_{o} g_{0} \alpha(t) \chi(\mathbf{r}),
$$


where $r_{o}$ represents the fixed ratio of the Clebsch-Gordan coefficients for the transitions $|g\rangle \rightarrow|e\rangle$ and $|s\rangle \rightarrow|e\rangle$.

To understand the basic idea of this method, let us first look at a very simplified picture by neglecting the coupling of the mode $a$ to the cavity output. The system is then described by the following simple Hamiltonian in the rotating frame (setting $\hbar=1$ ):

$$
H_{\text {sim }}=\Omega(\mathbf{r}, t) \sigma_{e g}+g(\mathbf{r}) a \sigma_{e s}+\text { H.c., }
$$

where $\sigma_{\mu \nu}=|\mu\rangle\langle\nu|(\mu, \nu=g, e, s)$ are the atomic transition operators, and H.c. stands for the Hermitian conjugate. The Hamiltonian $H_{\text {sim }}$ has the well-known dark state $|D\rangle$ (the instantaneous eigenstate with a zero eigenvalue) with the form [27]

$$
\begin{aligned}
|D\rangle & =\frac{1}{\sqrt{|g(\mathbf{r})|^{2}+|\Omega(\mathbf{r}, t)|^{2}}}[g(\mathbf{r})|g\rangle|0\rangle-\Omega(\mathbf{r}, t)|s\rangle|1\rangle] \\
& =\frac{1}{\sqrt{1+\left|r_{o} \alpha(t)\right|^{2}}}\left[|g\rangle|0\rangle-r_{o} \alpha(t)|s\rangle|1\rangle\right]
\end{aligned}
$$

where $|0\rangle$ and $|1\rangle$ represent the zero- and the one-photon state of the cavity mode $a$. Note that the dark state $|D\rangle$ actually only depends on the ratio between the parameters $g(\mathbf{r})$ and $\Omega(\mathbf{r}, t)$, so it becomes independent of the random atom position $\mathbf{r}$ with the interaction configuration specified above. If we start with the atom in the ground state $|g\rangle$, and gradually increase the Rabi frequency $\Omega(\mathbf{r}, t)$, under the adiabatic approximation, the system will remain in the dark state $|D\rangle$, which gradually evolves into the final state $|s\rangle|1\rangle$. Due to the independence of the state $|D\rangle$ on the variable $\mathbf{r}$, the relevant dynamics of this adiabatic evolution also becomes independent of the random atom site $\mathbf{r}$. This is the basic idea of the method to eliminate the influence of the randomness on the coupling coefficient $g(\mathbf{r})$.

Note that to make the dark state and the relevant dynamics independent of the random atom position $\mathbf{r}$, the driving pulse and the cavity mode need to have the same spatial mode structure. This is why the classical driving pulse is matched to the spatial mode of the cavity field, both along the cavity axis and transversely, which is routinely accomplished by way of illumination from one side mirror of the cavity. This configuration is different from the original proposals for adiabatic dynamics in cavity QED [27] in which the propagation direction of the driving pulse is perpendicular to the cavity axis with uniform illumination intensity. It is also distinct from the configuration employed in some recent interesting experiments directed toward achieving a singlephoton source $[17,18]$, which likewise employed uniform illumination transverse to the cavity axis and for which the atom is not localized axially. As a result, in these experiments some of the dynamics, such as the output pulse shape and phase, still depend on the unknown position of the atom, and are thus not fully controllable, as has been seen from the experiments.

We also would like to mention that in this configuration, the driving field and the quantized cavity output are collinear, and they need to be separated afterwards. The separation can be done through either polarization or frequency selection. This separation is actually pretty easy in the present case. In typical experimental configurations, the classical field drives one cavity mode from one cavity mirror (say 1) with a large detuning, and the single-photon quantum field together with some transmitted driving field are output from the other cavity mirror (say 2, with the transmission rate $t_{2}$ $>t_{1}$ ). Most of the driving field has been filtered already by the high-finesse cavity itself. The ratio between the intensities of the classical driving field and the quantum field output from side 2 is the same as their ratio inside the cavity, which does not need to be very large, since both of the atomic transitions are enhanced by the cavity and thus have comparable strength. As will be seen in the numerical simulations in Sec. III, inside the cavity, the driving field is typically assumed to be about five times stronger than the singlephoton field, and it is pretty easy to separate such a weak field with a polarization beam splitter at output side 2 .

To guarantee an adiabatic evolution, we need to fulfill the adiabatic condition, which means that the evolution time $T$ should be significantly longer than the frequency gap $\delta$ between the dark state and some other eigenstates of the Hamiltonian $H_{\text {sim }}$. The error probability due to the nonadiabaticity is estimated by $p_{a d}=1 /(\delta T)^{2}$. For the Hamiltonian $H_{\text {sim }}$, the frequency gap $\delta$ is given by $\delta=\sqrt{|g(\mathbf{r})|^{2}+|\Omega(\mathbf{r}, t)|^{2}}$. Thus, the adiabatic condition $\left[|g(\mathbf{r})|^{2}+|\Omega(\mathbf{r}, t)|^{2}\right] T^{2} \gg 1$ depends on the atom position $\mathbf{r}$. If the coupling coefficient $g(\mathbf{r})$ changes by a factor of 2 , the error probability $p_{a d}$ will change by a factor of 4 for the same evolution time $T$. However, if $T$ is sufficiently long, the error probability $p_{a d}$ remains small, and the relevant system dynamics will be still very insensitive to the randomness of the atom's position. To estimate $p_{a d}$, we can use the average value of the coupling rate $g(\mathbf{r})$.

In the above simple picture, we neglect the coupling of the mode $a$ to the cavity output. This is only a valid picture in the good-cavity limit with the evolution time $T \ll 1 / \kappa$, where $\kappa$ is the cavity decay rate. However, in practice, it is better to operate the system in the limit with $T \geqslant 1 / \kappa$. There are several advantages of operating the system in this limit: first, without the requirement $T \ll 1 / \kappa$, it is easier to satisfy the adiabatic condition for which $T$ should be sufficiently long; second, in this limit it is easier to modulate the Rabi frequency $\Omega(\mathbf{r}, t)$ by changing the intensity of the driving laser $\varepsilon(t)$ incident from one side mirror of the cavity. In this way, one can efficiently control the pulse shape of the cavity output by modulating the shape $\varepsilon(t)$ of the driving laser, which is useful for many applications. In the limit $T \geqslant 1 / \kappa$, we need to take into account, from the beginning, the coupling of the mode $a$ to the continuum cavity output, and the whole system will then have infinite levels. We will describe in the section this more involved interaction configuration. The above simple three-level picture, though it does not describe the real experimental configuration, does help in understanding the basic idea of the adiabatic method.

\section{B. Theoretical model and its approximate analytical solution}

Now we look at the more complicated theoretical model, which includes the coupling of the mode $a$ to the continuum 
cavity output. If we adiabatically apply a classical driving pulse $\varepsilon(t)$ as shown in Fig. 1, one photon will be emitted from the transition $|e\rangle \rightarrow|s\rangle$, and the cavity will output a single-photon pulse. We want to show below that this singlephoton pulse has a definite pulse shape which is independent of the randomness in the atom's position $\mathbf{r}$ and in the coupling rate $g(\mathbf{r})$. In this way, although the atom's position and the absolute value of the light-atom coupling rate are not fully controlled, we can nevertheless fully control the properties of the output single-photon pulse by modulating the driving laser pulse $\varepsilon(t)$. This is an important feature for many applications of this setup, which we will discuss in Sec. IV. There are several equivalent ways to describe the coupling of the mode $a$ to the continuum cavity output $[9,30,31]$. Since we want to calculate the output pulse shape within the adiabatic approximation, it is convenient to use the Hamiltonian approach [30,31]. The derivation here is similar to the calculation in Ref. [30] for the pulse shape from an ensemble of atoms. The whole Hamiltonian, including the coupling to the cavity output, has the following form in the rotating frame [31]:

$$
\begin{aligned}
H= & \left(\Delta-i \gamma_{s} / 2\right) \sigma_{e e}+\left[\Omega(\mathbf{r}, t) \sigma_{e g}+g(\mathbf{r}) a \sigma_{e s}+\text { H.c. }\right] \\
& +i \sqrt{\kappa / 2 \pi} \int_{-\omega_{b}}^{+\omega_{b}} d \omega\left[a^{\dagger} b(\omega)-a b^{\dagger}(\omega)\right] \\
& +\int_{-\omega_{b}}^{+\omega_{b}} d \omega\left[\omega b^{\dagger}(\omega) b(\omega)\right],
\end{aligned}
$$

where $b(\omega)$, with the standard commutation relation $\left[b(\omega), b^{\dagger}\left(\omega^{\prime}\right)\right]=\delta\left(\omega-\omega^{\prime}\right)$, denote the one-dimensional free-space modes that couple to the cavity mode $a$. We only need to consider the free-space modes within a finite bandwidth $\left[\omega_{s e}-\omega_{b}, \omega_{s e}+\omega_{b}\right]$ with the carrier frequency $\omega_{s e}$ $\left(\omega_{s e}\right.$ is the frequency splitting between the levels $|s\rangle$ and $|e\rangle)$, since all the modes outside of this bandwidth have negligible contributions to the dynamics due to the large detuning (larger than $\omega_{b}$ ). Within this bandwidth, the coupling between $b(\omega)$ and the cavity mode $a$ is approximately a constant, and we denote it by $\sqrt{\kappa / 2 \pi}$ for convenience, where $\kappa$ is the effective cavity decay rate, as we will see. The bandwidth $\omega_{b}$ should be chosen to be much larger than $\kappa$, but still much smaller than $\omega_{s e}$.

We have assumed that the driving laser and the cavity mode $a$ couple resonantly to the corresponding free-space atomic transitions. However, we emphasize that our scheme still works for the case of off-resonant coupling. By considering the off-resonant scheme, there is no win with respect to losses due to the atomic decay, since in this case the time scale also slows down. So it suffices here to consider the resonant coupling case. However, in the Hamiltonian (6), it is still helpful to include a single-photon-transition detuning $\Delta$ to account for the trapping potential difference for the levels $|g\rangle$ and $|e\rangle$ induced by the FORT beam (this potential is basically the same for the levels $|s\rangle$ and $|g\rangle$ for a FORT beam with linear polarization as in our current experiments). The potential difference between the level $|g\rangle$ and $|e\rangle$, in general, depends as well on the random atom position $\mathbf{r}$.
The imaginary part of the Hamiltonian (6) accounts for the spontaneous emission loss, where $\gamma_{s}$ denotes the total spontaneous emission rate of the upper level $|e\rangle$. In writing this form, we have assumed that the spontaneous emission photon escapes and that the atom after a spontaneous emission will not be repumped. This is a good assumption for the interesting region where the spontaneous emission loss is not big, and the atom thus has a very small probability to be repumped after emitting a spontaneous emission photon. As a result of this assumption, the spontaneous emission only contributes to the leakage error which is properly represented by Eq. (6) [32].

We treat the atom's position $\mathbf{r}$ in the Hamiltonian (6) as a classical stochastic variable, and neglect its quantum nature. This is a good approximation for the current experimental situation where the atom is still quite hot. There have been some analyses of the noise from quantum motion effects in high- $Q$ cavities with very cold atoms [33].

We start with the atom in the ground state $|g\rangle$, and then apply a classical driving pulse $\varepsilon(t)$. This pulse can efficiently control the time evolution of the Rabi frequency $\Omega(\mathbf{r}, t)$ in the Hamiltonian (6). To see this, we write the input-output equation for the cavity mode $a^{\prime}$ [31]

$$
\dot{a}^{\prime}=-i \omega_{s e} a^{\prime}-\frac{\kappa}{2} a^{\prime}-\sqrt{\kappa} a_{\mathrm{in}}^{\prime}(t),
$$

where $a_{\mathrm{in}}^{\prime}(t)$ is the field operator for the input driving pulse coupling to the mode $a^{\prime}$, with $\left\langle a_{\text {in }}^{\prime}(t)\right\rangle=\varepsilon(t)$ and $\left[a_{\text {in }}^{\prime}(t), a_{\text {in }}^{\prime \dagger}\left(t^{\prime}\right)\right]=\delta\left(t-t^{\prime}\right)$. By assumption, the mode $a^{\prime}$ has the same frequency as the mode $a$, which is resonant to the free-space atomic transition $|s\rangle \rightarrow|e\rangle$, so the eigenfrequency of $a^{\prime}$ is $\omega_{s e}$. Such a situation corresponds, for example, to the case of the $\left(a, a^{\prime}\right)$ modes of orthogonal polarization, but degenerate in frequency, although this is not an essential requirement. In Eq. (7), we have neglected the small depletion of $a^{\prime}$ caused by the coupling to the atomic transition $\sigma_{e g}$, since $a^{\prime}$ is driven by a strong classical pulse $\varepsilon(t)$ which dominates its time evolution. We write the mean values of $a^{\prime}$ and $\quad a_{i n}^{\prime}(t) \quad$ as $\quad\left\langle a^{\prime}\right\rangle=\alpha(t) e^{-i \omega_{g e} t} \quad$ and $\quad\left\langle a_{\mathrm{in}}^{\prime}(t)\right\rangle=\varepsilon(t)$ $=\widetilde{\varepsilon}(t) e^{-i \omega_{g e} t}$, where $\widetilde{\varepsilon}(t)$ is the slowly varying amplitude of the driving laser. From Eq. (7), we get a time evolution equation for the mean value $\alpha(t)$, which has the following immediate solution:

$$
\alpha(t)=\int_{0}^{t} \widetilde{\varepsilon}(\tau) e^{\left(i \omega_{g s}-\kappa / 2\right)(t-\tau)} d \tau
$$

The variation rate of $\widetilde{\varepsilon}(\tau)$ is characterized by the inverse of the operation time $T$ (the pulse duration), which is typically much smaller than the hyperfine frequency splitting $\omega_{g s}$ (about $9 \mathrm{GHz}$ for cesium atoms). Hence, a partial integration of Eq. (8) yields

$$
\alpha(t) \simeq \frac{\tilde{\varepsilon}(t)-e^{\left(i \omega_{g s}-\kappa / 2\right) t} \widetilde{\varepsilon}(0)}{-i \omega_{g s}+\kappa / 2}\left[1+o\left(\frac{1}{\omega_{g s} T}\right)\right] .
$$


We assume that $\tilde{\varepsilon}(t)$ gradually increases from zero with $\widetilde{\varepsilon}(0) \simeq 0$. Then, within a good approximation, we have $\alpha(t) \propto \tilde{\varepsilon}(t)$ from Eq. (9). In the following, without loss of generality, we assume $\alpha(t)$ to be real by choosing an appropriate constant phase of $\tilde{\varepsilon}(t)$. The time behavior of the Rabi frequency $\Omega(\mathbf{r}, t)$ is completely determined by $\alpha(t)$ [note that $\Omega(\mathbf{r}, t)=r_{o} g_{0} \alpha(t) \chi(\mathbf{r})$ from Eq. (3)], that is, by the amplitude $\widetilde{\varepsilon}(t)$ of the driving laser.

The dark state (5) can be rewritten as $|D\rangle=\cos \theta|g\rangle|0\rangle$ $-\sin \theta|s\rangle|1\rangle$, with $\cos \theta=1 / \sqrt{1+\left|r_{o} \alpha(t)\right|^{2}}$ independent of the atom's position $\mathbf{r}$. The state $|B\rangle$ complementary to the dark state is usually called the bright state with $|B\rangle=\sin \theta|g\rangle|0\rangle$ $+\cos \theta|s\rangle|1\rangle$. To solve the dynamics governed by the Hamiltonian (6), we can expand the state $|\Psi\rangle$ of the whole system into the following superposition:

$$
|\Psi\rangle=\left(c_{d}|D\rangle+c_{b}|B\rangle+c_{e}|e\rangle|0\rangle\right) \otimes|\mathrm{vac}\rangle+|s\rangle|0\rangle \otimes\left|\varphi_{1}\right\rangle,
$$

where $|v a c\rangle$ denotes the vacuum state of the free-space modes $b(\omega)$, and

$$
\left|\varphi_{1}\right\rangle=\int_{-\omega_{b}}^{+\omega_{b}} d \omega c_{\omega} b^{\dagger}(\omega)|\mathrm{vac}\rangle
$$

represents the state (not normalized) of the single-photon output pulse. The coefficients $c_{d}, c_{b}, c_{e}$, and $c_{\omega}$ in Eq. (10) are time dependent. At the time $t=0$, we have $c_{d}=1, c_{b}$ $=c_{e}=c_{\omega}=0$, and $\cos \theta=1$. After applying a classical driving pulse $\varepsilon(t), \cos \theta$ slowly changes with $\alpha(t)$, and we need to compute the time evolution of all the coefficients $c_{d}, c_{b}, c_{e}, c_{\omega}$ in Eq. (10) by substituting $|\Psi\rangle$ into the Schrödinger equation $i \partial_{t}|\Psi\rangle=H|\Psi\rangle$.

To go on with this task, let us first take the adiabatic approximation, which assumes the time derivative $\partial_{t} \cos \theta$ $\approx 0$. As a result, $\partial_{t}|D\rangle$ and $\partial_{t}|B\rangle$ become negligible. We will check the validity of the adiabatic approximation and calculate various nonadiabatic corrections in the following section through numerical methods. In the adiabatic limit, the populations in the bright state $|B\rangle$ and in the excited state $|e\rangle$ are negligible, so we assume $c_{b} \approx c_{e} \approx 0$. The coefficients $c_{d}$ and $c_{\omega}$ satisfy the following evolution equations:

$$
\begin{aligned}
& \dot{c}_{d}=-\sqrt{\kappa / 2 \pi} \sin \theta \int_{-\omega_{b}}^{+\omega_{b}} c_{\omega} d \omega, \\
& \dot{c}_{\omega}=-i \omega c_{\omega}+\sqrt{\kappa / 2 \pi} c_{d} \sin \theta .
\end{aligned}
$$

Equation (13) has the solution

$$
c_{\omega}(t)=\sqrt{\kappa / 2 \pi} \int_{0}^{t} e^{-i \omega(t-\tau)} c_{d}(\tau) \sin \theta(\tau) d \tau,
$$

which, substituted into Eq. (12), leads to

$$
\begin{aligned}
\dot{c}_{d} & =-\frac{\kappa}{2 \pi} \sin \theta \int_{0}^{t} \frac{\sin \left[\omega_{b}(t-\tau)\right]}{t-\tau} c_{d}(\tau) \sin \theta(\tau) d \tau \\
& \simeq-(\kappa / 2) c_{d} \sin ^{2} \theta .
\end{aligned}
$$

The approximation in Eq. (15) is valid since the bandwidth $\omega_{b}$ satisfies $\omega_{b} T \gg 1$, where the operation time $T$ characterizes the time scale for a significant change of $c_{d}$ and $\sin \theta$. Therefore, the dark-state coefficient $c_{d}$ satisfies the cavity free-decay equation, with the decay rate $\kappa$ replaced by the effective rate $\kappa \sin ^{2} \theta$. This can be easily understood since $\sin ^{2} \theta$ is the probability of the component $|s\rangle|1\rangle$ in the dark state $|D\rangle$, and it is exactly this component that couples to the cavity output. Equation (15) has the straightforward solution

$$
c_{d}=\exp \left(-\frac{\kappa}{2} \int_{0}^{t} \sin ^{2} \theta(\tau) d \tau\right) .
$$

We want to know the single-photon pulse shape $f(t)$ of the cavity output state $\left|\varphi_{1}\right\rangle$. Suppose now that $T$ is the final time of the interaction (i.e., the operation time determined by the driving laser pulse is from 0 to $T$ ). The pulse shape $f(t)$ is connected with the coefficients $c_{\omega}(t)$ before the frequency components in $\left|\varphi_{1}\right\rangle$ by the Fourier transformation [31]

$$
f(t)=\frac{1}{\sqrt{2 \pi}} \int_{-\omega_{b}}^{+\omega_{b}} d \omega c_{\omega}(T) e^{-i \omega(t-T)} .
$$

From Eqs. (14), (16), and (17), we finally obtain

$$
f(t)=\sqrt{\kappa} \sin \theta(t) \exp \left(-\frac{\kappa}{2} \int_{0}^{t} \sin ^{2} \theta(\tau) d \tau\right) .
$$

Note that the single-photon pulse shape $f(t)$ is completely determined by $\theta(t)$, i.e., by the driving pulse shape $\widetilde{\varepsilon}(t)$, and is independent of the random atom's position $\mathbf{r}$ and the absolute value of the coupling coefficient $g(\mathbf{r})$. As we have mentioned before, this is the main advantage of this adiabatic method compared with either the Raman scheme or prior proposals based upon adiabatic passages with uniform illumination $[17,18,27]$, and this feature is essential for many applications of this setup.

The above result is obtained within the adiabatic approximation, and in the adiabatic limit, the solution is independent of the atomic spontaneous emission rate $\gamma_{s}$ and the detuning $\Delta$. This is only a rough picture. In the following, we will solve exactly the dynamics governed by the Hamiltonian (6) without the use of the adiabatic approximation. The exact solution is necessary in the following two senses: first, we need to verify the above ideal picture and to find out under what condition this picture is approximately valid. Though in the three-level case, we have some simple estimation of the condition for the adiabatic following, it is not easy to figure out the exact adiabatic following condition for the more realistic situation of a continuum of external modes. In this case, the argument based on the level spacing is not valid. We need to know how long the operation time $T$ should be to satisfy the adiabatic following condition. We also expect that the atomic spontaneous emission cannot be made negligible simply by increasing the operation time $T$. Its rate $\gamma_{s}$ should be small enough to satisfy the strong-coupling condition $\kappa \gamma_{s} \ll \bar{g}^{2}$, where $\bar{g}$ denotes the average of the coupling rate $g(\mathbf{r})$ [34]. Second, in real experiments, the operation time $T$ 
is not infinitely long, and the coupling rate $\bar{g}$ cannot be arbitrarily larger than the decay rates $\kappa$ and $\gamma_{s}$ due to limitation of the technology (for instance, in Caltech experiments, typically, $\bar{g} / 2 \pi$ is around $20 \mathrm{MHz}$, and $\kappa / 2 \pi \sim \gamma_{s} / 2 \pi$ $\sim 6 \mathrm{MHz}$ ). In this case, there would be various nonadiabatic corrections to the above ideal picture, for instance, the atom may go down from the level $|e\rangle$ to $|s\rangle$ through a spontaneous emission, and then we lose the emitted photon and thus have no output from the cavity; or we have a single-photon output, but it is in a wrong and unknown pulse shape due to its sensitivity to the random atom position induced by the nonadiabatic contributions. It is desirous and important to calculate quantitatively the magnitudes of these noises to predict the real experiments. The exact solution of the system dynamics is only available with the numerical methods, which is the main task of the following section.

\section{EXACT NUMERICAL SIMULATIONS}

\section{A. The numerical calculation method}

In this section, we solve exactly the system dynamics governed by the Hamiltonian (6) through numerical simulations, and calculate various nonadiabatic corrections and noise magnitudes. For numerical simulations of the Hamiltonian (6), we need to discretize the free-space field $b(\omega)$ by introducing a finite but small frequency interval $\delta \omega$ between two adjacent modes. Then, in total we have about $N$ $\approx 2 \omega_{b} / \delta \omega$ free-space modes, with the $j$ mode denoted by $b_{j}$. The frequency detuning $\omega_{j}$ of the $j$ mode is given by $\omega_{j}=(j-N / 2) \delta \omega$. To assure that there is no change of the physical result after the discretization, we should choose the frequency interval $\delta \omega$ much smaller than the inverse of the operation time $T$, and the bandwidth $\omega_{b}$ much larger than the cavity decay rate $\kappa$.

For the numerical simulation, we can similarly expand the state $|\Psi\rangle$ of the whole system in the form of Eq. (9), with the single-photon pulse state replaced by

$$
\left|\varphi_{1}\right\rangle=\sum_{j=1}^{N} c_{j} b_{j}^{\dagger}|\mathrm{vac}\rangle .
$$

From the Hamiltonian (6), we get the following complete set of equations for the coefficients $c_{d}, c_{b}, c_{e}$, and $c_{j}$ :

$$
\begin{gathered}
\dot{c}_{d}=-\dot{\theta} c_{b}-\kappa^{\prime} \sin \theta \sum_{j=1}^{N} c_{j}, \\
\dot{c}_{b}=\dot{\theta} c_{d}-i \sqrt{\Omega^{2}(\mathbf{r}, t)+g^{2}(\mathbf{r})} c_{e}+\kappa^{\prime} \cos \theta \sum_{j=1}^{N} c_{j}, \\
\dot{c}_{e}=\left(-i \Delta-\gamma_{s} / 2\right) c_{e}-i \sqrt{\Omega^{2}(\mathbf{r}, t)+g^{2}(\mathbf{r})} c_{b}, \\
\dot{c}_{j}=-i(j-N / 2) \delta \omega c_{j}+\kappa^{\prime} \sin \theta c_{d}-\kappa^{\prime} \cos \theta c_{b},
\end{gathered}
$$

where the effective decay rate $\kappa^{\prime} \equiv \sqrt{\kappa \delta \omega / 2 \pi}$. We obtain the solutions of these coefficients by numerically integrating Eqs. (20)-(23) from the time $t=0$ to $t=T$, where $T$ is the

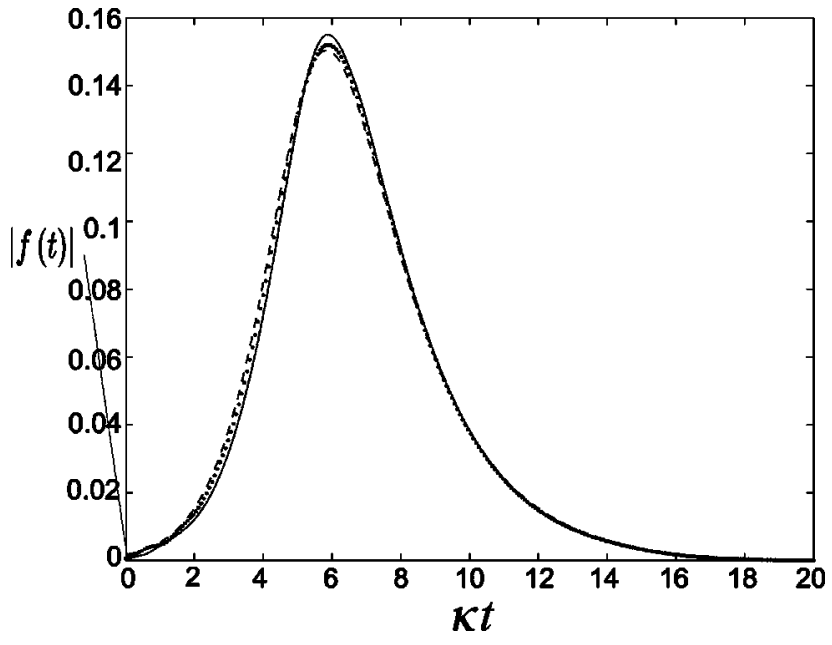

FIG. 2. The shape of the output single-photon pulse described by the amplitude $|f(t)|$ vs the time $t$ for the coupling rates $g(\mathbf{r})$ $=3 \kappa$ (solid curve) and $g(\mathbf{r})=6 \kappa$ (dotted curve). The dashed curve represents the pulse shape in the ideal adiabatic limit calculated from Eq. (16). In this figure, we have taken $\gamma_{s}=\kappa, \Delta=0$, and $T$ $=20 / \kappa$. The driving pulse $\tilde{\varepsilon}(t)$ is in a Gaussian shape with the peak at $t=T / 2$ and a width $t_{w}=T / 5$.

duration of the driving pulse $\widetilde{\varepsilon}(t)$. We assume that $\widetilde{\varepsilon}(t)$ is a Gaussian pulse so that $\alpha(t)$ is a Gaussian function of the time $t$, with its peak value at $T / 2$, and a width $t_{w}$ significantly smaller than $T / 2$. All the functions of $\theta$ in Eqs. (20)-(23) are decided from $\cos \theta=1 / \sqrt{1+\left|r_{o} \alpha(t)\right|^{2}}$ and $\sqrt{\Omega^{2}(\mathbf{r}, t)+g^{2}(\mathbf{r})}$ $=g(\mathbf{r}) / \cos \theta$. To simulate the randomness of the atom position $\mathbf{r}$, we vary the value of $g(\mathbf{r})$ in the simulation to look at whether the final result changes with this variation.

\section{B. Shape of the output single-photon pulse}

The output single-photon pulse shape $f(t)$ can be easily constructed from the solution of the coefficients $c_{j}$ through a discrete version of Eq. (17). The result is shown in Fig. 2 for $g(\mathbf{r})=3 \kappa$ and $g(\mathbf{r})=6 \kappa$. Although we have not made definitive measurements, we estimate that $g(\mathbf{r})$ varies within a factor of roughly 2 in the current Caltech experiment $[2,3,16]$. Here and in the following, the pulse-shape function $f(t)$ is always renormalized according to $\int|f(t)|^{2} d t=1$ for convenience of comparison. We see that the two curves overlap very well, which confirms the prediction that the output pulse shape is very insensitive to the randomness of the coupling coefficient $g(\mathbf{r})$ when the adiabatic condition is satisfied (we take $T=20 / \kappa$ for this figure). We also draw in this figure the pulse shape $f(t)$ given by Eq. (18) derived in the ideal adiabatic limit, which agrees well with the exact numerical results. Therefore, within the adiabatic condition, we can use the analytical result (18) to design the shape of the output single-photon pulse by modulating the driving pulse shape $\tilde{\varepsilon}(t)$.

\section{Noise magnitudes and the adiabatic condition}

To quantify the noise magnitudes in this setup, we can define several error probabilities. First, we have the leakage 
error due to the atomic spontaneous emission. A photon may be emitted to modes other than the principal cavity mode through the spontaneous emission with the rate $\gamma_{s}$. As a result, the norm $\left|c_{d}\right|^{2}+\left|c_{b}\right|^{2}+\left|c_{e}\right|^{2}+\sum_{j=1}^{N}\left|c_{j}\right|^{2}$ of the state (10) decays with the time $t$, and we can use

$$
P_{\text {spon }}=1-\left|c_{d}(T)\right|^{2}-\left|c_{b}(T)\right|^{2}-\left|c_{e}(T)\right|^{2}-\sum_{j=1}^{N}\left|c_{j}(T)\right|^{2}
$$

at the final time $T$ to quantify the total possibility of the spontaneous emission loss. Second, due to the finiteness of the operation time $T$ and the pumping field amplitude $\widetilde{\varepsilon}(t)$, the initial excitation in the dark state is not necessarily fully transferred to the output quantum signal at the final time, and we can use

$$
P_{\text {tran }}=\left|c_{d}(T)\right|^{2}+\left|c_{b}(T)\right|^{2}+\left|c_{e}(T)\right|^{2}
$$

at the time $T$ to quantify the transmission inefficiency. In principle, we can arbitrarily decrease the transmission inefficiency by increasing the duration $T$ or the amplitude $\widetilde{\varepsilon}(t)$ of the pumping field. Finally, even if a photon is emitted into the cavity output field, it is not necessarily in the right pulse shape as given by Eq. (18) due to the nonadiabatic correction. This nonadiabatic correction depends on the random atom position and is unknown, so it is also a source of noise. To quantify this noise, we denote the ideal pulse shape given in Eq. (18) as $f_{\text {id }}(t)$, and the real pulse shape calculated from the numerical simulation as $f_{\text {real }}(t)$, then the shape mismatching error can be described by

$$
P_{\text {mis }}=\left|1-\frac{\int_{0}^{T} f_{\text {real }}^{*}(t) f_{\text {id }}(t) d t}{\left[\int_{0}^{T}\left|f_{\text {real }}(t)\right|^{2} d t \int_{0}^{T}\left|f_{\text {id }}(t)\right|^{2} d t\right]^{1 / 2}}\right| .
$$

This quantity is directly related to the visibility of the fringes if we interfere two single-photon pulses from two such setups.

For the example shown in Fig. 2, with $g(\mathbf{r})=3 \kappa=3 \gamma_{s}$ (the other parameters are given in the figure caption), we have $P_{\text {spon }} \approx 4.0 \%, P_{\text {tran }} \approx 0.04 \%, P_{\text {mis }} \approx 0.18 \%$. The dominant source of noise is the leakage error $P_{\text {spon }}$ induced by the spontaneous emission. If we increase the operation time $T$ so that the adiabatic condition is better satisfied, the abovedefined noise magnitudes can be reduced a little bit, but not too much. For instance, with the above example but with $T$ $=30 / \kappa$, we have $P_{\text {spon }} \approx 3.33 \%$ and $P_{\text {mis }} \approx 0.15 \%$. On the other hand, if $T$ is reduced so that the adiabatic condition is not well satisfied, the error probabilities can significantly increase. Figure 3 shows the output pulse shapes for $g(\mathbf{r})$ $=3 \kappa$ and $g(\mathbf{r})=6 \kappa$ with $T=5 / \kappa$. The two curves are obviously different from each other and are also different from the ideal shape as given by Eq. (18). For the example with $g(\mathbf{r})=3 \kappa=3 \gamma_{s}$ and $T=5 / \kappa$, we have $P_{\text {spon }} \approx 36 \%, P_{\text {tran }}$ $\approx 3.2 \%, P_{\text {mis }} \approx 2.7 \%$. All the noise magnitudes significantly increase. In particular, the spontaneous emission loss be-

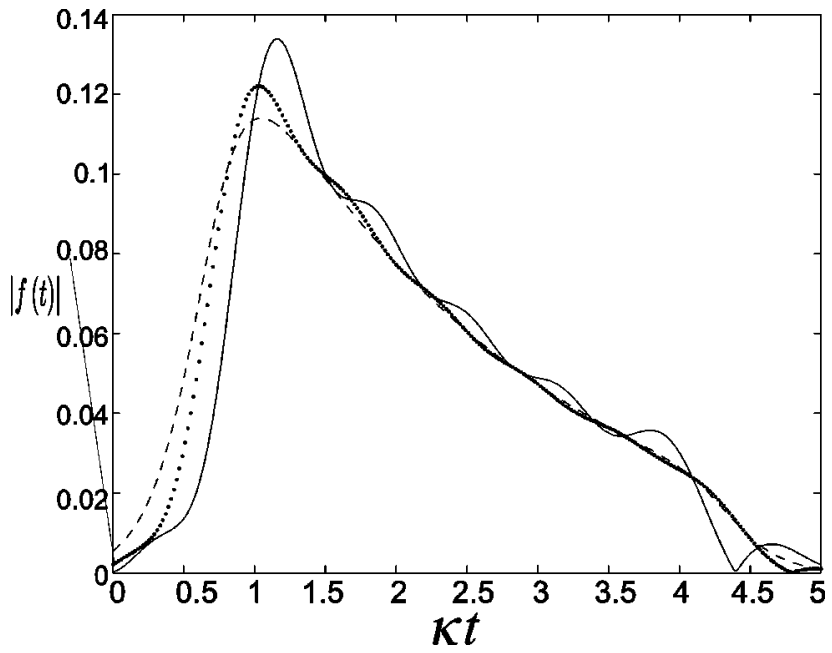

FIG. 3. The shape $|f(t)|$ of the output single-photon pulse for the coupling rates $g(\mathbf{r})=3 \kappa$ (solid curve), $g(\mathbf{r})=6 \kappa$ (dotted curve), and in the ideal adiabatic limit (dashed curve). We assumed the same condition as in Fig. 2, except that $T=5 / \kappa$, which does not satisfy well the adiabatic condition.

comes very big. This can be easily understood since without the adiabatic condition, the excited state $|e\rangle$ will be populated during the operation, and thus we have a correspondingly larger spontaneous emission loss.

\section{The strong-coupling condition}

Next we look at the requirement of the strong-coupling condition. Let $\bar{g}$ denote the average value of the coupling rate $g(\mathbf{r})$. Normally one requires $\bar{g}^{2} \gg \kappa \gamma_{s}$ to satisfy the strong-coupling condition. We can define the strong-coupling parameter $d_{s c}$ as $d_{s c}=\bar{g}^{2} / \kappa \gamma_{s}$, and calculate the abovedefined noise magnitudes $P_{\text {spon }}, P_{\text {tran }}, P_{\text {mis }}$ under different values of the parameter $d_{s c}$. We assumed $T=30 / \kappa$ and $\Delta$ $=0$ in the calculation so that the adiabatic condition is well satisfied. It turns out that the spontaneous emission loss $P_{\text {spon }}$ is always the dominant loss (about ten times larger than other sources of noise). Thus, in Fig. 4, we only show the calculation result for $P_{\text {spon }}$ under different values of $d_{s c}$. The result can be approximately simulated by an empirical curve with $P_{\text {spon }} \approx 1 /\left(4 d_{s c}\right)$.

We can use this simple formula to estimate the spontaneous emission loss under different experimental conditions. Actually, in current experiments, the strong-coupling condition is only marginally satisfied. For instance, for the cesium atom in the Caltech group, $\left(\kappa, \gamma_{s}\right) / 2 \pi \approx(8,5.2) \mathrm{MHz}$ (note that $\kappa$ and $\gamma_{s}$ here denote the energy decay rates, which are two times the corresponding amplitude decay rates) [2,3], and $\bar{g} / 2 \pi$ is expected to be $\approx 15 \mathrm{MHz}$ for the transition $\left(6 S_{1 / 2}, F=4, m=+4\right) \rightarrow\left(6 P_{3 / 2}, F=4, m=+4\right) \quad[$ Note that the transition $\left(6 S_{1 / 2}, F=4, m=+4\right) \rightarrow\left(6 P_{3 / 2}, F=5, m=\right.$ $+5)$ cannot be used as a $\Lambda$ configuration though it has a slightly larger coupling rate $\bar{g}$ ]. These values lead to $d_{s c}$ $=\bar{g}^{2} / \kappa \gamma_{s} \approx 5.4$ and a resulting spontaneous emission loss around $4.6 \%$, which is quite accessible with the present tech- 


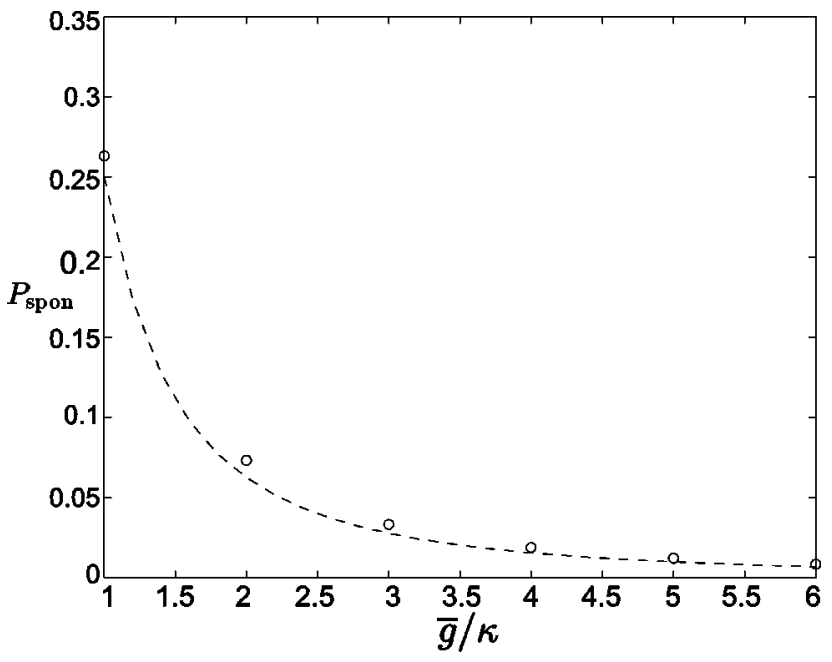

FIG. 4. The spontaneous emission loss $P_{\text {spon }}$ vs the average coupling rate $\bar{g}$ (in the units of the cavity decay rate $\kappa$ ). We assumed $\Delta=0$ and $\gamma_{s}=\kappa$, so the strong-coupling parameter $d_{s c}$ is simply $(\bar{g} / \kappa)^{2}$. The circles represent the results from the numerical calculation, and the dashed curve is from the empirical formula $P_{\text {spon }} \approx 1 /\left(4 d_{s c}\right)$ which simulates well the numerical results.

nology. As another example, in the recent experiment [18], one has $\left(\kappa, \gamma_{s}\right) / 2 \pi \approx(1.25,6.0) \mathrm{MHz}$ and $\bar{g} / 2 \pi \approx 2.5 \mathrm{MHz}$ according to the estimation there. With these parameters, $\bar{g}^{2} / \kappa \gamma_{s} \approx 0.83$ and we estimate that the spontaneous emission loss is about $P_{\text {spon }} \approx 30 \%$ if one uses the scheme here. If the usual adiabatic scheme is adopted with a uniform driving pulse perpendicular to the cavity axis, the spontaneous emission loss should be still significantly larger, as will be seen from the simulation in the last section.

\section{E. The influence of the single-photon transition detuning}

In the above calculations, we assumed $\Delta=0$. Finally, we discuss the influence of a nonzero single-photon detuning $\Delta$. In Fig. 5, we show the calculation result of the exact pulseshape function $f_{\text {real }}(t)$ with a significant detuning $\Delta=\kappa$, and compare it with the ideal pulse shape function $f_{\text {id }}(t)$ given by Eq. (18) for both the amplitude and the phase. The other parameters for this example are given in the figure caption. From the figure, we see that the two amplitudes $\left|f_{\text {real }}(t)\right|$ and $\left|f_{\text {id }}(t)\right|$ still overlap very well, but their phases become a bit different due to the detuning.

This phase difference is determined by the the detuning $\Delta$, whereas the latter depends on the different level shift between ground and excited states, and hence varies with the atom position within the FORT beam. In the case of the simple level scheme depicted in Fig. 1, the states $|g\rangle$ and $|e\rangle$ would have spatially dependent level shifts of opposite sign, which would lead to variations in $\Delta$ comparable to the trap depth. Fortunately, there is a simple way to mitigate this difficulty by considering the multilevels involved for the FORT beam, as described in Ref. [35], so that the trapping potentials for the states $|g\rangle$ and $|e\rangle$ are very nearly the same. For example, for the experiment of Ref. [16], the difference in trap depth for $|g\rangle$ and $|e\rangle$ is roughly $10 \%$ of the trap

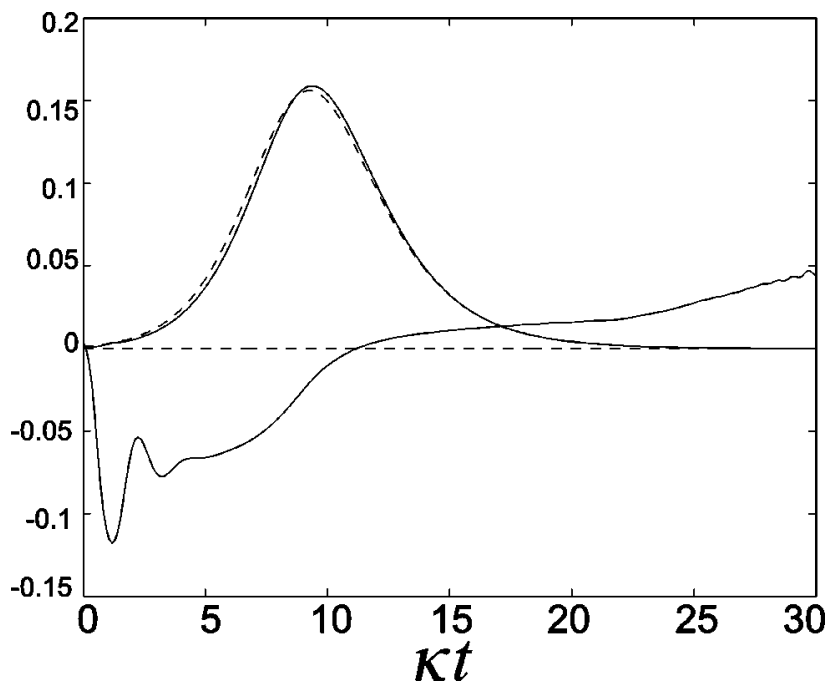

FIG. 5. The amplitude (the modulus) and the phase (divided by $\pi / 2$ ) of the real pulse shape $f_{\text {real }}(t)$ (two solid curves) and the ideal pulse shape $f_{\text {id }}(t)$ (two dashed curves) vs the time $t$ with the singlephoton transition detuning $\Delta=\kappa$. We assumed $g(\mathbf{r})=3 \kappa, \gamma_{s}=\kappa$, and $T=30 / \kappa$. In this case, the main difference between $f_{\text {real }}(t)$ and $f_{\text {id }}(t)$ lies in the phase difference.

depth. Relative to the current analysis, there is then a variation in $\Delta$ as the atom moves in the FORT potential, which is unknown when the adiabatic protocol is implemented. The curve in Fig. 5 is an attempt to estimate the impact of such random detunings by setting $\Delta=\kappa$, which exceeds the actual magnitude of any spatially dependent detunings for FORT depths up to about $50 \mathrm{MHz}$. The phase difference in the pulse-shape function caused by the unknown detunings is a source of noise, which contributes to the shape mismatching error defined in Eq. (26). For this example with $g(\mathbf{r})=3 \kappa$, we have $P_{\text {spon }} \approx 3.33 \%, P_{\text {tran }} \approx 10^{-4}$, which are basically the same as the corresponding case without detuning, but $P_{\text {mis }}$ $\approx 3.33 \%$, which becomes significantly larger due to the contribution of the phase difference.

\section{F. Comparison with the usual adiabatic scheme}

In our scheme, the driving pulse is matched to a cavity mode which has basically the same spatial mode, structure as the cavity-QED light. In usual adiabatic schemes [18,27], the driving laser is assumed to be perpendicular to the cavity axis with uniform illumination intensity. We expect that with the present interaction configuration, our scheme is more insensitive to the randomness in the atom position. To compare the two configurations more quantitatively, we have calculated the output pulse shapes and noise magnitudes for both schemes.

First, let us assume that the atom has been trapped in one potential well, but the coupling rate $g(\mathbf{r})$ may vary within a factor of 2 due to the unknown atom's position. In Fig. 6, we show the calculation results of the output pulse shapes. The solid curve shows the pulse-shape function $|f(t)|$ when $g(\mathbf{r})=3 \kappa$ and $\Omega_{m}(\mathbf{r})=15 \kappa$, where $\Omega_{m}(\mathbf{r})$ is the maximum of $\Omega(\mathbf{r}, t)$ with respect to time $t[\Omega(\mathbf{r}, t)$ is assumed to be a Gaussian function of $t$ as specified in the caption of Fig. 2]. 


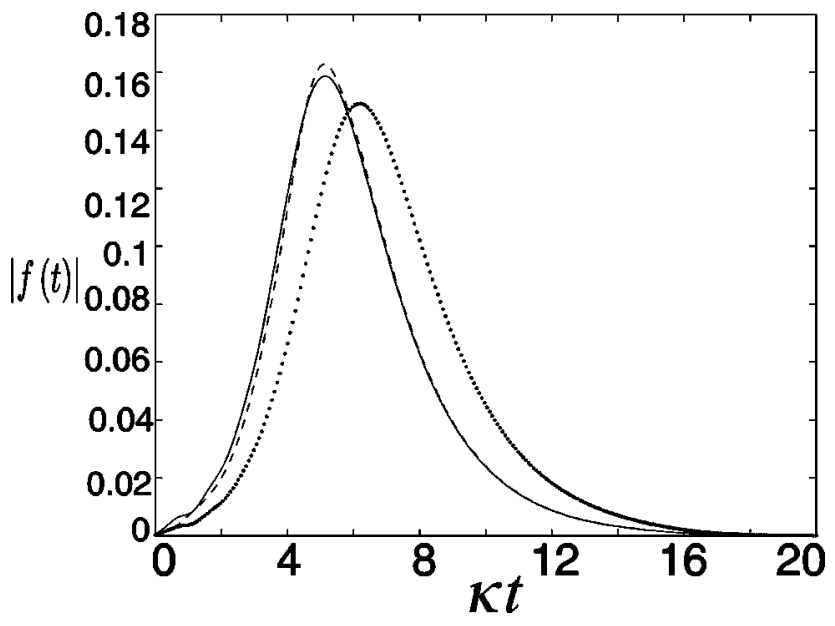

FIG. 6. The shape $|f(t)|$ of the output single-photon pulse for the following pairs of coupling rates and the driving Rabi frequencies: first, $g(\mathbf{r})=3 \kappa$ and $\Omega_{m}(\mathbf{r})=15 \kappa$ (solid curve); second, $g(\mathbf{r})$ $=6 \kappa$ and $\Omega_{m}(\mathbf{r})=30 \kappa$ (dashed curve), and finally, $g(\mathbf{r})=6 \kappa$ and $\Omega_{m}(\mathbf{r})=15 \kappa$ (dotted curve). The other parameters are the same as in Fig. 2.

Now, if $g(\mathbf{r})$ varies by a factor of 2 due to change of the atom's position, in our scheme the Rabi frequency will correspondingly change by the same ratio. The dashed curve shows the pulse shape for $g(\mathbf{r})=6 \kappa$ and $\Omega_{m}(\mathbf{r})=30 \kappa$. One can see that the two curves overlap very well with the mode mismatching noise smaller than $0.2 \%$. In contrast, in usual adiabatic schemes with uniform illumination intensity, $\Omega_{m}(\mathbf{r})$ does not change as $g(\mathbf{r})$ varies with the atom position, so we have the same $\Omega_{m}(\mathbf{r})=15 \kappa$. The dotted curve in Fig. 6 shows the pulse shape for $g(\mathbf{r})=6 \kappa$ and $\Omega_{m}(\mathbf{r})$ $=15 \kappa$. It is significantly different from the above two curves with a notable mode mismatching noise $P_{\text {mis }} \approx 6.9 \%$. The improvement by this scheme would become more impressive if $g(\mathbf{r})$ has a larger variation, both in its magnitude and in its sign. If $g(\mathbf{r})$ gets a random sign as the atom goes through different axial positions of the cavity, in the usual adiabatic scheme, the pulse-shape function $f(t)$ will also pick up a random sign. However, in the present scheme, this random sign in $f(t)$ can be eliminated. Therefore, by this interaction configuration, the scheme is more robust to the random variation of the atom's position.

The improvement by this protocol is also very remarkable if we consider the case where the atom is not fixed in one potential well, and may move from well to well in the axial direction. The variation of the atom's position in the axial direction is typically fast compared with the operation time $T$, so we have a time-varying atom position $\mathbf{r}$ and coupling rate $g(\mathbf{r})$. Here, we consider an explicit form of the time variation of $g(\mathbf{r})$ by assuming $g(\mathbf{r}(t))=6 \kappa \sin \left(4 \pi t / T+\varphi_{0}\right)$, where the phase $\varphi_{0}$ is randomly chosen corresponding to the randomness in the initial atom's position. It is enough to illustrate the general result by considering this special example. First, let us calculate the output pulse shape $f(t)$ for the usual adiabatic scheme, where $\Omega_{m}(\mathbf{r})$ is fixed as a constant $[17,18,27]$. The solid and the dash-dotted curves in Fig. 7 show the real parts of $f(t)$ with initial phase $\varphi_{0}=0$ and

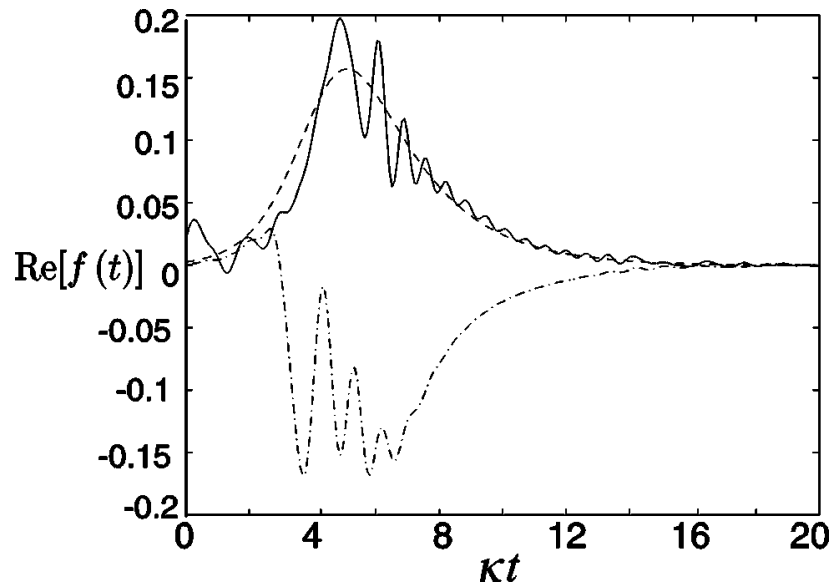

FIG. 7. The real part of the pulse-shape function $\operatorname{Re}[f(t)]$ as $g(\mathbf{r})$ varies with time in the form $g(\mathbf{r})=6 \kappa \sin \left(4 \pi t / T+\varphi_{0}\right)$ in the usual adiabatic scheme with $\varphi_{0}=0$ (solid curve) and $\varphi_{0}=\pi / 2$ (dash-dotted curve), respectively. The dashed curve shows the ideal pulse shape calculated from Eq. (18). The other parameters in this figure are the same as those in Fig. 2.

$\varphi_{0}=\pi / 2$, respectively [the imaginary parts of $f(t)$ are actually small and negligible]. The two curves do not overlap at all. Neither the magnitude nor the phase of the pulse shape $f(t)$ can be controlled with this scheme. We also calculate the spontaneous emission loss $P_{\text {spon }}$ for this example. The average spontaneous emission loss is about $P_{\text {spon }} \approx 25 \%$.

Similarly, we can calculate the pulse shape for the same example with the present scheme. In this case, due to the atomic motion, $\Omega_{m}(\mathbf{r})$ varies with time in the same way as $g(\mathbf{r})$, but the ratio $\Omega_{m}(\mathbf{r}) / g(\mathbf{r})$ is kept constant. Figure 8 shows the real part of the shape function $f(t)$ in this case, with the solid and the dash-dotted curves corresponding to the initial phase $\varphi_{0}=0$ and $\varphi_{0}=\pi / 2$, respectively. Although the two curves do not overlap very well, they still look similar with the same phase. They also roughly agree with the ideal shape function given by Eq. (18), which is shown as the dashed curve in Fig. 8. The average mode mismatching noise

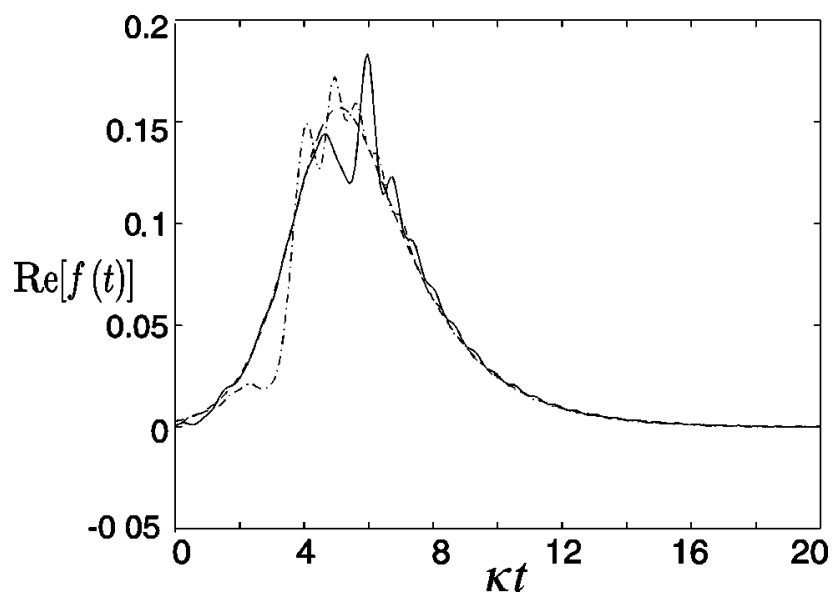

FIG. 8. The real part of the pulse-shape function $\operatorname{Re}[f(t)]$ calculated for the same example as in Fig. 7, but now for the present adiabatic scheme where the driving Rabi frequency varies in the same way as the the coupling rate when the atom moves. 
for these two curves is given by $P_{\text {mis }} \approx 1.1 \%$, and the average spontaneous emission loss is $P_{\text {spon }} \approx 9.4 \%$. The spontaneous emission loss is also significantly reduced with the present scheme. This can be understood as follows: if one has a constant $\Omega_{m}(\mathbf{r})$ as the usual adiabatic scheme, when the atom moves to the place with $g(\mathbf{r})$ near to zero, the adiabatic condition is not well satisfied, and as a result, one has a considerably large spontaneous emission loss; however, in the present scheme, in the place where $g(\mathbf{r})$ is near zero, $\Omega_{m}(\mathbf{r})$ is also near zero. The excitation probability of the atom is then reduced, and the adiabatic condition is better satisfied. Consequently, one has a smaller spontaneous emission loss.

\section{APPLICATIONS}

There have been many proposals to use the setup with single atoms in high- $Q$ cavities for various applications, such as for the single-photon or entangled-photon source [6,7], for quantum communication between different cavities [9], for atomic quantum teleportation $[11,12]$, and for quantum computation [13]. In these proposals, one always assumed that the atom is well localized so that the Lamb-Dicke condition is satisfied. However, one can apply the method here to all of the schemes mentioned above, to eliminate the challenging Lamb-Dicke condition. Basically, what one needs to do is to replace the Raman scheme with the adiabatic scheme, and to keep the pumping laser collinear with the cavity axis, so that the driving pulse and the cavity mode have the same spatial mode structure. All the calculation results (for the noise magnitudes, pulse shape, etc.) in this paper apply to these schemes. After the improvement, it becomes considerably easier to implement these schemes with the current technology. Here, we briefly review these schemes and discuss how to incorporate the present method into these schemes.

\section{A. Controllable single-photon or entangled-photon source}

It is desirable to have a single-photon source with all its properties fully controllable, including its emission direction, emission time, and pulse shape. This kind of source has important applications in some recent quantum-information processing schemes [36], which are normally based on the interference of different single-photon pulses. To get interference between different single-photon pulses, one requires all the pulses to be directional and have the same time shape. Recently, there have been significant experimental advances in the realization of the single-photon source [17,18,37-39]. In the experiments based on the solid-state material [37-39], the single-photon emitter has a fixed position, and one can, in principle, control the pulse shape well. However, the emitted pulse is typically not directional. On the other hand, in current experiments $[17,18]$ with high- $Q$ cavities, the emitted pulse is directional, but its shape is not well controlled since with uniform illumination of a perpendicular driving pulse, the waveform $f(t)$ depends on the time history of the coupling rate $g(\mathbf{r})$, which in turn depends on the atom's position. As the atom falls through the cavity, it has basically a random trajectory, leading to unknown variations in $g(\mathbf{r})$ both in magnitude and sign. It is a challenging experimental endeavor to demonstrate a single-photon source with all the properties mentioned above fully controllable.

The method in this paper shows that the single atom trapped in a high- $Q$ cavity is a good candidate for the realization of the fully controllable single-photon source. Though the coupling rate $g(\mathbf{r})$ is not completely fixed in current setups due to the difficulty in fully localizing the atom, the emitted single-photon pulse has a definitely wellcontrollable time shape and emission direction with an appropriate design of the interaction configuration as has been shown before.

As shown in Ref. [7], with a more involved atomic level structure, it is possible to engineer entanglement between different single-photon pulses. It is straightforward to combine the method here with that scheme to eliminate the requirement of the Lamb-Dicke condition in Ref. [7] so that one can get an entangled single-photon source with the "hot" trapped atom as well.

\section{B. Quantum communication between different cavities}

The dynamics governed by the Hamiltonian (6) is reversible if we neglect the atomic spontaneous emission $\gamma_{s}$. Therefore, if one directs the emitted single-photon pulse back to the cavity, and at the same time reverses both of the time shapes of the single-photon pulse and the driving pulse, the single-photon pulse will be completely absorbed as long as the noise effects are negligible. It was first proposed in Ref. [9] that one can use this kind of a phenomenon to achieve quantum communication between different cavities, that is, to transfer quantum states of a trapped atom from one cavity to another cavity. For this purpose, one can require that the emitted single-photon pulse has a time-symmetric shape by modulating the driving pulse shape. For a timesymmetric pulse, its time reversal is itself, so we can directly input this pulse to another cavity with the same configuration but with a time-reversed driving pulse, then the singlephoton pulse will be completely absorbed by this cavity, which transfers the atomic state from one cavity to the other one. The scheme in Ref. [9] is based on the Raman configuration, but it is straightforward to transfer it to the adiabatic configuration discussed in this paper so that it works with a hot trapped atom. Note that the same setup can also be used for storage of a single-photon pulse with a known shape $[30,40,41]$.

To get a time-symmetric single-photon pulse for a complete absorption of the second cavity, Ref. [9] gives a numerical solution to the shape of the driving pulse. For the adiabatic configuration, one has an analytic expression (18) which connects the shape of the output single-photon pulse to the shape of the driving pulse, and this expression has been verified to be a good approximation under reasonable experimental parameters in Sec. III through the exact numerical calculations. With this analytical expression, it becomes easier to design the shape $\widetilde{\varepsilon}(t)$ of the driving pulse. The form of $\sin \theta(t)$ can be easily solved from Eq. (18) (see also Ref. [40]) with the expression 


$$
\sqrt{\kappa \sin \theta(t)}=\frac{f(t)}{\sqrt{1-\int_{0}^{t} f^{2}(\tau) d \tau}} .
$$

The form of $\sin \theta(t)$ is immediately available from this equation for any desirable output pulse shape $f(t)$ (which has been assumed to be real and positive for simplicity). Then, the shape of the driving pulse can be easily decided from $\widetilde{\varepsilon}(t) \propto \alpha(t)$ and $\sin \theta(t)=r_{o} \alpha(t) / \sqrt{1+\left|r_{o} \alpha(t)\right|^{2}}$, where $r_{o}$ is the ratio of the Clebsch-Gordan coefficients. For instance, if we want to have a time-symmetric $f(t)$ in the period $0 \leqslant t$ $\leqslant T$ with the form $f(t)=\sqrt{\beta / 2} \operatorname{sech}[\beta(t-T / 2)]$, where we have assumed $\operatorname{sech}(-\beta T / 2) \ll 1, \sin \theta(t)$ should be in the form $\sin \theta(t)=\sqrt{\beta / \kappa} \sqrt{1+\tanh [\beta(t-T / 2)]}$. Note that we only have a solution of $\theta(t)$ when the rate $\beta<\kappa / 2$, which is consistent with the observation that any pulse from the decay of a cavity cannot vary with time faster than the cavity decay rate. From $\sin \theta(t)$, we see that the shape $\tilde{\varepsilon}(t)$ of the driving pulse should be chosen according to

$$
\widetilde{\varepsilon}(t) \propto \sqrt{\frac{1+\tanh [\beta(t-T / 2)]}{(\kappa / \beta-1)-\tanh [\beta(t-T / 2)]}} .
$$

As a special case, if $\kappa / \beta=2, \widetilde{\varepsilon}(t) \propto e^{\beta(t-T / 2)}$, which grows exponentially with the time $t$ for the operation period $0 \leqslant t$ $\leqslant T$. Therefore, we have a simple solution to the driving pulse shape for quantum communication between two different cavities: for the first cavity, we apply an exponentially increasing pulse with $\widetilde{\varepsilon}(t)=\widetilde{\varepsilon}(0) e^{\kappa t / 2}$, and for the second cavity we apply its time reversal, that is, an exponentially decreasing pulse with the decay rate $\kappa / 2$. The pulse duration $T$ should satisfy $\kappa T \gg 1$, and the initial value $\widetilde{\varepsilon}(0)$ is determined by the requirement $r_{o} \alpha(T / 2)=1$. The single-photon pulse connecting the two cavities then has a time-symmetric shape with $f(t) \propto \operatorname{sech}[\kappa(t-T / 2) / 2]$.

\section{Entanglement generation and atomic quantum teleportation}

If one has two cavities, each with an atom inside, one can maximally entangle these two atoms 1 and 2 by the following method: The two atoms are initially prepared in the state $|g\rangle$, and then we excite them to the state $|s\rangle$ with a small possibility $p_{0} \approx 1-\exp \left[-\kappa \int_{0}^{T} \sin ^{2} \theta(\tau) d \tau\right]$ through an incomplete adiabatic passage. The output pulses from the two cavities, each with a mean photon number $p_{0}$, have a definite pulse shape as we have shown before, so that they can interfere with each other at a 50\%-50\% beam splitter. The outputs of the beam splitter are detected by two single-photon detectors, and if we register a photon from one of the detectors, due to the interference, we do not know from which cavity the registered photon comes. The two atoms 1 and 2 are thus projected to a quantum superposition state $\left(|g\rangle_{1}|s\rangle_{2}\right.$ $\left.\pm|s\rangle_{1}|g\rangle_{2}\right) / \sqrt{2}$, which is maximally entangled. The method described here is just an adiabatic passage version of the scheme in Refs. [11,12]. By transformation from the Raman version to the adiabatic passage version, the output pulse shapes become insensitive to the random atom's position as is required for interference, which is important for the scheme to work with hot atoms.

After entanglement has been generated, one can use it for atomic Bell inequality detection, for quantum teleportation of atomic states [12], or even for realization of quantum repeaters [42]. To realize quantum repeaters, what one needs to do is to simply replace the atomic ensemble in the scheme in Ref. [42] by the setup of a single atom in a high- $Q$ cavity.

For the above applications, in addition to the entanglement generation, we also need to do some single-bit operations. These single-bit operations should also be performed in a suitable way so that they are insensitive to the random atom position $\mathbf{r}$. One way is to still use adiabatic passages. It is possible to realize any single-bit operation with adiabatic passages $[43,44]$, but for this purpose one needs to use a four-level scheme instead of the $\Lambda$ configuration. There is actually a simpler way for getting robust single-bit operations based on the Raman transitions. Note that for single-bit operations, we do not need to use any cavity mode or cavity effect. We can shine two traveling-wave beams on the atom coupling to the transitions $|g\rangle \rightarrow|e\rangle$ and $|s\rangle \rightarrow|e\rangle$. They are assumed to be collinear and propagating along the $x$ axis, which is perpendicular to the cavity axis $z$. The two traveling-wave beams are broad with the beam radius much larger than the typical variation length of the atom's position. With this condition, the two Rabi frequencies for the transitions $|g\rangle \rightarrow|e\rangle$ and $|s\rangle \rightarrow|e\rangle$ are given by $\Omega_{1}(\mathbf{r})$ $=\Omega_{10} e^{i \omega_{g} e^{x / c}}$ and $\Omega_{2}(\mathbf{r})=\Omega_{20} e^{i \omega_{s} e^{x / c}}$, respectively, where $\Omega_{10}$ and $\Omega_{20}$ are basically independent of the atom position r. Under a large detuning $\Delta$, the effective Raman coupling rate $\Omega_{R} \sim \Omega_{1}(\mathbf{r}) \Omega_{2}^{*}(\mathbf{r}) / \Delta \propto e^{i \omega_{g s} x / c}$ is very insensitive to the random atom's position $\mathbf{r}$, since $c / \omega_{g s}$ is typically much larger than the variation length of the position. Therefore, as long as we do not need to use the cavity effect, a Raman scheme with two broad collinearly propagating beams suffices to eliminate the sensitivity to the random atom's position.

\section{Quantum computation}

In principle, we can also use this setup for quantum computation [13], and eliminate the requirement of the LambDicke condition by performing all the quantum gates using adiabatic passages $[43,45]$ with appropriate configurations. However, the requirements for a universal quantum computation are more challenging compared with the applications mentioned above, and this is somewhat a long-term goal, so we do not discuss here the details of this possibility.

\section{DISCUSSION OF THE EXPERIMENTAL SITUATION}

Finally, let us mention the current experimental situation related to this work at the Caltech group. In the Caltech experiment, a single cesium atom is trapped inside the highfinesse cavity with a FORT beam. The atomic states $|g\rangle,|s\rangle$, and $|e\rangle$ correspond to the hyperfine levels $\left(6 S_{1 / 2}, F=3, m=\right.$ $+3),\left(6 S_{1 / 2}, F=4, m=+4\right)$, and $\left(6 P_{3 / 2}, F=4, m=+4\right)$, re- 
spectively. The FORT beam is incident on one of the cavity mirrors and resonant to a longitudinal mode of the cavity. Presently, the FORT wavelength $\lambda_{\mathrm{FORT}}$ is $936 \mathrm{~nm}$. This wavelength was chosen because with such a beam, the trapping potentials for the ground $6 S_{1 / 2}$ manifold and the excited $6 P_{3 / 2}$ manifold are nearly identical. Considering only this reduced manifold of states, we find that the expression for the FORT potential of the ground states $|g\rangle$ and $|s\rangle$ is given [46] by

$$
U_{F O R T}(\mathbf{r})=\frac{\pi c^{2} \gamma_{s}}{2 \omega_{0}^{3}}\left(\frac{2}{\Delta_{2}}+\frac{1}{\Delta_{1}}\right) I(\mathbf{r}) .
$$

Here, $\Delta_{1}\left(\Delta_{2}\right)$ is the detuning of the FORT light of frequency $\omega_{\mathrm{FORT}}=2 \pi c / \lambda_{\mathrm{FORT}}$ from the $P_{1 / 2}\left(P_{3 / 2}\right)$ level, and $\gamma_{s} / 2 \pi \approx 5.2 \mathrm{MHz}$ is the spontaneous decay rate of the level $6 P_{3 / 2}$. The intensity $I(\mathbf{r})$ of the standing-wave mode inside the cavity is given by

$$
I(\mathbf{r})=\frac{8 P}{\pi w_{0}^{2}} \sin ^{2}\left(\frac{2 \pi z}{\lambda_{\mathrm{FORT}}}\right) \exp \left(-\frac{x^{2}+y^{2}}{w_{0}^{2}}\right),
$$

where $w_{0} \approx 25 \mu \mathrm{m}$ is the waist of the Gaussian mode, and $P$ is the power of the FORT beam inside the cavity. The trap frequencies $\nu_{\text {axial }}, \nu_{\text {radial }}$ in the axial and radial directions follow from these expressions as

$$
\begin{aligned}
& \left(\nu_{\text {axial }}, \nu_{\text {radial }}\right) \\
& =\frac{1}{2 \pi \hbar}\left(\sqrt{2 U_{0} \frac{\hbar^{2} \omega_{F O R T}^{2}}{m c^{2}}}, \sqrt{2 U_{0} \frac{\hbar^{2}}{m\left(w_{0}\right)^{2}}}\right),
\end{aligned}
$$

where $U_{0}=U_{F O R T}(\mathbf{0})$ is the trap depth. The typical power of the FORT beam measured outside the cavity is about $1 \mathrm{~mW}$, and the power $P$ inside the cavity is enhanced by a factor of the cavity finesse, which is about 2200 at the wavelength of the FORT beam. With this number, the typical values for the trap depth and frequencies are given by $U_{0} \approx 38 \mathrm{MHz}$, $\nu_{\text {axial }} \approx 510 \mathrm{kHz}$, and $\nu_{\text {radial }} \approx 4.3 \mathrm{kHz}$, respectively. The current achievable temperature $T_{\text {tem }}$ of the trapped atom is a significant fraction of the trap depth $U_{0}$ (such as a half). With such a temperature, the spatial extent of the atomic motion in the axial and radial directions are estimated, respectively, by

$$
\begin{gathered}
\delta z / \lambda_{\mathrm{FORT}} \approx(1 / 2 \pi) \arcsin \sqrt{k_{B} T_{\mathrm{tem}} / U_{0}}, \\
\delta r_{\perp} \approx w_{0} \sqrt{-\ln \left(1-k_{B} T_{\mathrm{tem}} / U_{0}\right)},
\end{gathered}
$$

which will induce significant variation of the coupling rate $g(\mathbf{r})$ given by Eq. (1). For example, for the temperature of half of the trap depth, the axial uncertainty is $120 \mathrm{~nm}$, while the radial one is $15 \mu \mathrm{m}$. These uncertainties cause variations in $g$ of $30 \%$ due to the radial motion, and $35 \%$ due to the axial one. Therefore, within the current experimental technique, it is important to use the method given in this paper to make the application schemes insensitive to the variation of $g(\mathbf{r})$. The time scale for the variation of $g(\mathbf{r})$ is estimated by the inverse of the trap frequencies $\nu_{\text {axial }}$ and $\nu_{\text {radial }}$ in the axial and radial directions, respectively. The operation time $T$ is typically significantly shorter than $1 / \nu_{\text {radial }}$, but longer or comparable to $1 / \nu_{\text {axial }}$. So, we can take the static average of $g(\mathbf{r})$ in the radial direction, and the dynamical average of $g(\mathbf{r})$ in the axial direction as discussed in Ref. [34].

We also would like to note that although the method in this paper shows that many application schemes of the cavity-QED setup can be demonstrated before the achievement of efficient cooling of the trapped atom inside the cavity, the cooling is still an important and desirable technology yet to be achieved to significantly increase the trapping time of the atom. In addition, a combination of the cooling technology and the method here could further improve the performance of various application schemes.

\section{SUMMARY}

In summary, we have shown that the setup with a single trapped atom in a high- $Q$ cavity can be used to realize many cavity-QED and quantum-information processing schemes even if the atom is still hot and not fully localized in space (the Lamb-Dicke condition is not yet satisfied). This could significantly simplify the on-going experiments, since it means many interesting schemes can be demonstrated with the present technology before the achievement of efficient cooling inside the cavity. Even with further advances in atomic localization in cavity-QED, our scheme should lead to a greater robustness against certain experimental nonidealities. The basic idea of this method is to design an appropriate adiabatic passage so that the relevant dynamics only depend on the ratio of two coupling rates. Though each of the coupling rates is sensitive to the unknown or timevarying atom's position, their ratio is fixed and controllable, as the two rates depend on the random atom position in the same way with the appropriate interaction configuration that we have described. We confirm the validity of this method by solving the complete model, which describes the realistic setup. The approximate analytical solution and the exact numerical simulations agree with each other. From the numerical simulations, we also calculate quantitatively various noise magnitudes in this setup, and show that one can achieve reasonably good performance with the values of the parameters based on the present technology. Finally, we show that this method can be incorporated into many previous schemes, allowing the demonstration of these application schemes without the requirement of the full localization of the atom.

\section{ACKNOWLEDGMENTS}

L.-M.D. thanks Axel Kuhn for discussions. This work was supported by the Caltech MURI Center for Quantum Networks under ARO Grant No. DAAD19-00-1-0374, by the National Science Foundation under Grant No. EIA-0086038, and by the Office of Naval Research. L.-M.D. also acknowledge support from the Chinese Science Foundation, Chinese Academy of Sciences, and the national "97.3" project. 
[1] For a review, see contributions in the special issue of Phys. Scr., T 76, 127 (1998).

[2] J. Ye, D.W. Vernooy, and H.J. Kimble, Phys. Rev. Lett. 83, 4987 (1999).

[3] J. McKeever and H.J. Kimble, in Laser Spectroscopy: XIV International Conference, edited by $\mathrm{S}$. Chu and J. Kerman (World Scientific, Singapore, 2002).

[4] C.J. Hood et al., Science 287, 1447 (2000).

[5] P.W.H. Pinkse, T. Fischer, T.P. Maunz, and G. Rempe, Nature (London) 404, 365 (2000).

[6] C.K. Law and H.J. Kimble, J. Mod. Opt. 44, 2067 (1997).

[7] K.M. Gheri et al., Phys. Rev. A 58, R2627 (1998).

[8] A.C. Doherty, T.W. Lynn, C.J. Hood, and H.J. Kimble, Phys. Rev. A 63, 013401 (2000).

[9] J.I. Cirac, P. Zoller, H.J. Kimble, and H. Mabuchi, Phys. Rev. Lett. 78, 3221 (1997).

[10] S.J. Enk, J.I. Cirac, and P. Zoller, Science 279, 205 (1998).

[11] C. Cabrillo, J.I. Cirac, P. G.-Fernandez, and P. Zoller, Phys. Rev. A 59, 1025 (1999).

[12] S. Bose, P.L. Knight, M.B. Plenio, and V. Vedral, Phys. Rev. Lett. 83, 5158 (1999).

[13] T. Pellizari, S.A. Gardiner, J.I. Cirac, and P. Zoller, Phys. Rev. Lett. 75, 3788 (1995).

[14] G.R. Guthöhrlein, M. Keller, K. Hayasaka, W. Lange, and H. Walther, Nature (London) 414, 49 (2001).

[15] J. Eschner, Ch. Raab, F. Schmidt-Kaler, and R. Blatt, Nature (London) 413, 495 (2001)

[16] J. McKeever, J. Buck, A. Kuzmich, H.-C. Naegerl, D.M. Stamper-Kurn, and H.J. Kimble (unpublished).

[17] M. Hennrich, T. Legero, A. Kuhn, and G. Rempe, Phys. Rev. Lett. 85, 4872 (2000).

[18] A. Kuhn, M. Hennrich, and G. Rempe, Phys. Rev. Lett. 89, 067901 (2002).

[19] P. Horak, G. Hechenblaikner, K.M. Gheri, H. Stecher, and H. Ritsch, Phys. Rev. Lett. 79, 4974 (1997).

[20] S.J. van Enk, J. McKeever, H.J. Kimble, and J. Ye, Phys. Rev. A 64, 013407 (2001).

[21] H.W. Chan, A.T. Black, and V. Vuletic, e-print quant-ph/0208100.

[22] A.C. Doherty, A.S. Parkins, S.M. Tan, and D.F. Walls, Phys. Rev. A 56, 833 (1997); T.A.B. Kennedy and P. Zhou ibid. 64, 063805 (2001).

[23] H. Mabuchi, J. Ye, and H.J. Kimble, Appl. Phys. B: Lasers Opt. 68, 1095 (1999).

[24] Reference [20] does offer some suggestions for operationally discriminating the atomic position (determined by the FORT potential minima) relative to the cavity-QED field.

[25] In principle, they can be also the same mode.

[26] K. Bergmann, H. Theuer, and B.W. Shore, Rev. Mod. Phys. 70, 1003 (1998).

[27] A.S. Parkins et al., Phys. Rev. A 51, 1578 (1995).

[28] A. Sorensen and K. Molmer, Phys. Rev. Lett. 82, 1971 (1999).

[29] J.F. Poyatos, J.I. Cirac, and P. Zoller, Phys. Rev. Lett. 81, 1322 (1998).

[30] M. Fleischhauer, S.F. Yelin, and M.D. Lukin, Opt. Commun. 179, 395 (2000).
[31] D.F. Walls and G.J. Milburn, Quantum Optics (SpringerVerlag, Berlin, 1994).

[32] A leakage error means that after a spontaneous emission, the system goes out of the Hilbert space spanned by the state (10) with all the possible coefficients, since in this case one has a photon in a spontaneous emission mode.

[33] A.C. Doherty, A.S. Parkins, S.M. Tan, and D.F. Walls, J. Opt. B: Quantum Semiclassical Opt. 1, 475 (1999).

[34] More accurately, by the notation $\bar{g}$, we should specify the averaging method. For instance, in the Caltech experiment with an atom trapped by a FORT beam (see Sec. V for a discussion of the current experimental situation), the atom oscillates fast in the axial direction with an oscillation period typically smaller than the operation time $T$, so we should take the time average (the dynamical average) of $g(\mathbf{r})$ in the axial direction for each round of the driving pulse. The atom also oscillates in the radial direction, but the period is normally significantly longer than the operation time $T$. So, we can assume a fixed (unknown) radial position for each driving pulse, and this position changes for different rounds of the pulses. We then take an average (the static average) of the results over many rounds of the driving pulses. We have performed both kinds of averaging in the numerical simulation in Sec. III, and without surprise, they basically give the same result. If the temperature of the atomic motion is around half of the trapping potential as is the typical case in the Caltech experiment, the value of $\bar{g}$ from the accurate averaging is also close to the magnitude from some simple estimation, for instance, one can estimate it as the average of the minimum and the maximum $g(\mathbf{r})$. Hence, in the following, we will use the notation $\bar{g}$ without elaboration on the averaging methods, since the result is not sensitive to them.

[35] H.J. Kimble, C.J. Hood, T.W. Lynn, H. Mabuchi, D.W. Vernooy, and J. Ye, in Laser Spectroscopy: XIV International Conference, edited by Rainer Blatt et al. (World Scientific, Singapore, 1999), p. 80.

[36] E.g., E. Knill, R. Laflamme, and G.J. Milburn, Nature (London) 409, 46 (2001).

[37] J. Kim, O. Benson, H. Kan, and Y. Yamamoto, Nature (London) 397, 500 (1999).

[38] P. Michler et al., Science 290, 2282 (2000).

[39] Z. Yuan et al., Science 295, 102 (2002).

[40] M.D. Lukin, S.F. Yelin, and M. Fleischhauer, Phys. Rev. Lett. 84, 4232 (2000).

[41] L.-M. Duan, J.I. Cirac, and P. Zoller (unpublished).

[42] L.-M. Duan, M. Lukin, J.I. Cirac, and P. Zoller, Nature (London) 414, 413 (2001)

[43] L.M. Duan, J.I. Cirac, and P. Zoller, Science 292, 1695 (2001).

[44] R.G. Unanyan, B.W. Shore, and K. Bergmann, Phys. Rev. A 59, 2910 (1999).

[45] A. Recati, T. Calarco, P. Zanardi, J.I. Cirac, and P. Zoller, e-print quant-ph/0204030; B. Tregenna, A. Beige, and P.L. Knight, Phys. Rev. A 65, 032305 (2002), and references therein; J. Pachos, and H. Walther, e-print quant-ph/0111088.

[46] S.J.M. Kuppens, K.L. Corwin, K.W. Miller, T.E. Chupp, and C.E. Wieman, Phys. Rev. A 62, 013406 (2000). 